NASA Technical Memorandum 104499

AIAA-91-2370

\title{
An Experimental Trace Gas Investigation of Fluid Transport and Mixing in a Circular- to-Rectangular Transition Duct
}

B.A. Reichert and W.R. Hingst

Lewis Research Center

Cleveland, Ohio

and

T.H. Okiishi

Iowa State University

Ames, Iowa

Prepared for the

27th Joint Propulsion Conference

cosponsored by the AIAA, SAE, ASME, and ASEE

Sacramento, California, June 24-27, 1991 


\title{
AN EXPERIMENTAL TRACE GAS INVESTIGATION OF FLUID TRANSPORT AND MIXING IN A CIRCULAR-TO-RECTANGULAR TRANSITION DUCT
}

\author{
B. A. Reichert ${ }^{*}$, W. R. Hingst ${ }^{\dagger}$ \\ NASA Lewis Research Center, Cleveland, Ohio \\ T. H. Okiishi ${ }^{\ddagger}$ \\ Iowa State University, Ames, Iowa
}

\begin{abstract}
An ethylene trace gas technique was used to map out fluid transport and mixing within a circular-to-rectangular transition duct. Ethylene gas was injected at several points in a cross stream plane upstream of the transition duct. Ethylene concentration contours were determined at several cross stream measurement planes spaced axially within the duct. The flow involved a uniform inlet flow at a Mach number level of 0.5. Statistical analyses were used to quantitatively interpret the trace gas results. Also, trace gas data were considered along with aerodynamic and surface flow visualization results to ascertain transition duct flow phenomena. Convection of wall boundary layer fluid by vortices produced regions of high total pressure loss in the duct. The physical extent of these high loss regions is governed by turbulent diffusion.
\end{abstract}

\section{Nomenclature}

$\begin{array}{lll}C & = & \text { mean concentration defined by Eq. } \\ & = & \text { fluctuating concentration in Eq. (4) } \\ c^{\prime} & =\begin{array}{l}\text { mean concentration coefficient } \\ \text { defined by Eq. (3) }\end{array} \\ C^{*} & =\begin{array}{l}\text { duct inlet diameter } \\ D\end{array} & \text { turbulent diffusion coefficients } \\ & \text { (tensor notation) } \\ D_{i j} & = & \text { molecular diffusion coefficient in Eq. } \\ D_{m} & (4) \\ n & = & \text { exponent in Eq. (1) } \\ r & = & \text { radial distance from duct centerline } \\ r_{y}, r_{z} & = & \text { radii in Eq. (1) }\end{array}$

*Aerospace Engineer, Inlet, Duct, and Nozzle Flow Physics Branch, Member AIAA

${ }^{\dagger}$ Aerospace Engineer, Inlet, Duct, and Nozzle Flow Physics Branch, Member AIAA.

${ }^{\ddagger}$ Professor and Chair, Mechanical Engineering Department, Member AIAA

Copyright @ 1991 by the American Institute of Aeronautics and Astronautics, Inc. No copyright is asserted in the United States under Title 17, U.S. Code. The U.S. Government has a royalty-free license to exercise all rights under the copyright claimed herein for Governmental purposes. All other rights are reserved by the copyright owner.

$\begin{array}{lll}S & =\text { injection source strength in Eq. (7) } \\ U, V, W & =\text { mean velocity components } \\ U_{i} & =\text { mean velocity components (tensor } \\ u^{\prime}, v^{\prime}, w^{\prime} & =\text { notation) } \\ u_{i}^{\prime} & =\text { fluctuating velocity components } \\ x, y, z & =\text { (tensor notation) in Eq. (4) } \\ \eta & =\text { molar amount in Eq. (2) } \\ \mu_{y}, \mu_{z} & =\text { mean values defined by Eq. (12) } \\ \sigma_{y}^{2}, \sigma_{z}^{2} & =\text { variances defined by Eq. (13) } \\ \sigma_{y z} & =\text { covariance defined by Eq. (14) }\end{array}$

Introduction

TONAXISYMMETRIC exhaust nozzles are employed on high performance aircraft to improve performance. For instance, the Pratt and Whitney twodimensional/convergent-divergent nozzle described by Stevens et al. ${ }^{1}$ is a rectangular nozzle designed to allow thrust vectoring, thrust reversal, jet area variation, and expansion area variation. Reduced thermal emission is another benefit attributed to rectangular exhaust nozzles. Rectangular exhaust nozzles require a circularto-rectangular transition duct to connect the engine and nozzle.

Previous investigators have made detailed flow field surveys within circular-to-rectangular transition ducts using pressure probes, hotwire probes and laser anemometry. ${ }^{2-7}$ These results indicate the dominant feature within this flow field are pairs of vortices which appear downstream of the transition region of the duct near the diverging sidewalls. Associated with these vortices are regions of substantial total pressure loss.

For our investigation, a trace gas technique was used to determine fluid transport and mixing within a circularto-rectangular transition duct. The trace gas technique involves injecting and tracking a nonreacting discernible foreign gas in the host flow field being investigated. Information about the host flow field is acquired by measuring the concentration of the trace gas in the host gas at many locations downstream of the point of injection. The trace gas technique is a valuable tool for several reasons; it is relatively inexpensive and easy to use, and it is a source of Lagrangian flow field data. 
Our implementation of the trace gas technique involved injecting ethylene gas at seven different locations within a cross stream plane upstream of the transition duct and measuring the resulting flow field concentration of ethylene at four cross stream measurement planes spaced axially within the duct. Twenty-eight different planer concentration distributions were measured. All measurements were made with uniform incoming flow and an inlet Mach number of 0.50 . Some modifications were required to adapt the ethylene trace gas technique to the high speed flows involved in this study. Novel methods were employed to inject ethylene trace gas in the transition duct flow field with minimal disturbances and to sample the flow with virtually unlimited spatial resolution of the measurement locations in a cross stream plane within the transition duct.

Statistical analyses were used to quantitatively interpret the trace gas results. In addition, the results of a detailed aerodynamic survey (e.g. flow velocity magnitude and direction), free stream turbulence measurements and surface oil film visualization were considered with trace gas measurements to ascertain duct flow phenomena. Location, and to some extent the shape of the trace gas concentration contours is governed by convection of the trace gas by the mean flow. The amount of mixing or spreading of the contours is governed by turbulent diffusion of the trace gas. Convection of boundary layer fluid by vortices produced regions of lower total pressure levels (high loss). The physical extent of these high loss regions is governed by turbulent diffusion.

\section{Experimental Facilities}

\section{Circular-to-Rectangular Transition Duct}

Fig. 1 shows the lower half of the circular-torectangular transition duct and the Cartesian coordinate system used throughout this paper. The $x$-axis is coincident with the duct centerline and the $y$ - and $z$-axes are parallel to the major and minor axes of the duct exit. All coordinates have been normalized with the duct inlet diameter, which was $20.42 \mathrm{~cm}$. In the $y z$-plane through each cross section, the surface of the duct satisfies Eq. (1) where the parameters $r_{y}, r_{z}$ and $n$ vary with the axial distance $x$ and specify the exact cross-sectional shape.

$$
\left(\frac{y}{r_{y}}\right)^{n}+\left(\frac{z}{r_{z}}\right)^{n}=1
$$

In the circular cross section region $(n=2)$, at $x / D \leq 1.0$, the duct maintains a constant circular cross section. The duct cross section is constant and nearly rectangular in the rectangular cross section region $(n=$ 10 ), at $x / D \geq 2.5$. The cross-sectional shape of the transition duct becomes more rectangular as the value of is never truly rectangular. The duct cross section changes from circular to nearly rectangular in the transition region, from $1.0 \leq x / D \leq 2.5$. The aspect ratio of the duct, defined as the ratio of the major and minor axes lengths in the rectangular cross section region, is 3.0. The transition region length-to-diameter ratio is 1.5 .

The cross-sectional areas in the circular and rectangular cross section regions are equal. In the transition region the cross-sectional area increases to 1.15 times the area in the circular and rectangular cross section regions. The distribution of cross-sectional area in the transition region is as it would be if a duct with the same aspect ratio and transition region length-to-diameter ratio were constructed of circular and flat sections of metal only. This type of construction was believed to be representative of fabrication methods used in the aviation industry.

The duct is symmetric with respect to reflections across the horizontal $x y$ - and vertical $x z$-planes. For flow without inlet swirl the flow field retained the duct's two planes of symmetry. That is, any scaler flow field property, $f$, satisfied the condition $f(x, y, z)=f(x,-y, z)=$ $f(x, y,-z)=f(x,-y,-z)$. Thus, measurements in only one quadrant of the duct were necessary. Measurements were made in two adjacent quadrants, however, in the upper half of the duct on either side of the $x z$-plane, to confirm that the measured flow was symmetric.

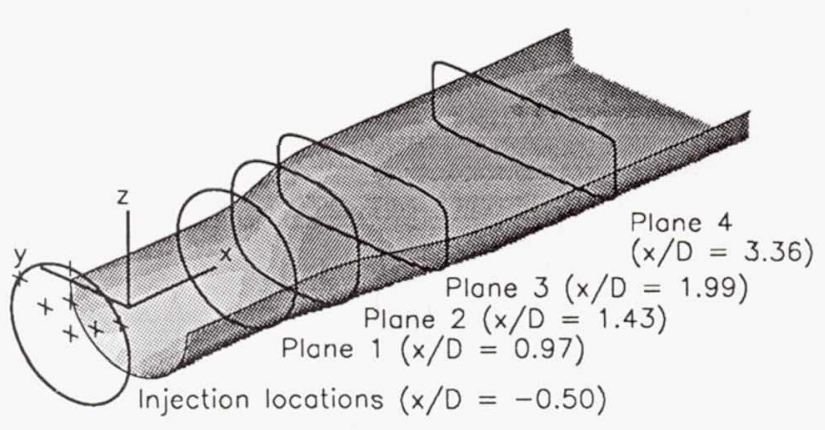

Fig. 1 Circular-to-rectangular transition duct.

\section{Test Facility}

All transition duct experiments were conducted at the NASA Lewis Research Center using the Internal Fluid Mechanics Facility. This facility was designed to support tests of a variety of internal flow configurations. For the experiment reported here, air proceeded from the test cell through a large settling chamber containing a honeycomb and two screens, and thence through an axisymmetric contraction having an area ratio of 57:1. From the contraction, the flow passed through a straight pipe to allow a boundary layer to develop then into the transition duct. After passing through the transition duct the flow was exhausted into a discharge plenum 
which was continuously evacuated by central compressor facilities.

Trace Gas Measurement Equipment

In this study, ethylene was used as the trace gas. Ethylene possesses several properties which make it desirable for trace gas studies:

(1) The molecular weight of ethylene, 28.1, is nearly the same as air, 29.0. This eliminates the effect of buoyancy forces on the motion of the trace gas.

(2) Under normal circumstances, ethylene and other hydrocarbon gases are only found in very minute quantities in air. Thus, the problem of having to adjust the trace gas concentration measurements to compensate for the background level of trace gas in air is avoided.

(3) The mean concentration of ethylene, or any hydrocarbon gas, can be measured very accurately with a flame ionization detector. This instrument is described later in this section.

The equipment necessary to make ethylene trace gas measurements in an air flow can be divided into three major components; the ethylene trace gas injection system, the flow field sampling system and the ethylene concentration measuring system. The relationship between these three major components and their subcomponents is shown in Fig. 2. Because there are no commercially available ethylene trace gas equipment items specifically intended for aerodynamic tests like those presently described, the equipment must be assembled from components designed for other applications.

\section{Ethylene Trace Gas Injection System}

The ethylene trace gas injection system was designed to introduce the ethylene gas into the flow field at a point location with a specific flow rate as unobtrusively as possible. The ethylene injection probe was designed to minimize the downstream flow disturbance from the probe while being sufficiently sturdy to withstand the aerodynamic forces of flows with Mach numbers as great as 0.5 . Further, the probe had to be capable of injecting trace gas at an adequate rate to allow detection throughout the downstream flow field.

The most common trace gas probe injection method previously used was an L-shaped probe, like a downstream facing total pressure probe. Several studies that have employed the ethylene trace gas technique have included the results of experiments designed to measure and minimize disturbances from L-shaped type injection probes. $^{8-12}$ In these studies the diameter of the injection probes ranged from $0.56 \mathrm{~mm}$ to $3.2 \mathrm{~mm}$. The results of these studies indicate that in regions of low turbulence intensity some minor probe disturbance is unavoidable

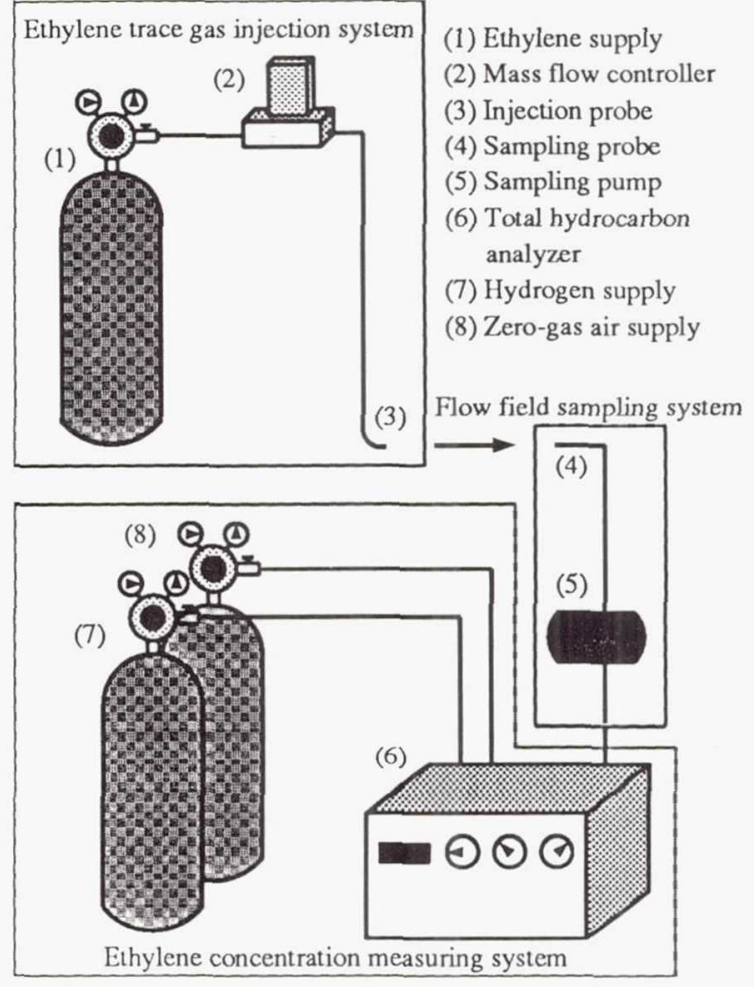

Fig. 2 Trace gas measurement equipment schematic.

and the smaller probes produced less trace gas spreading. However, in regions of higher turbulence intensity, the disturbance created by the probe is not of significant magnitude compared to the existing turbulence to materially effect the mixing of the trace gas.

All of the ethylene trace gas injection probes described in the cited literature were operated in comparatively low speed flows and utilized a cantilever design. For the transition duct study presently considered a probe design change was necessary. For the injection probe to be as small as possible and yet withstand the aerodynamic loading imposed when it is extended to the center of the duct free stream at Mach numbers as great as 0.50 required abandoning the cantilever design of previously used probes. Instead, a catenary design was used as shown in Fig. 3. The injection tube was supported along its full length by a second tube which was held in tension across a rigid frame. The cross section of the rigid frame and the wall bounding the flow are shown in Fig. 3. The ethylene trace gas injection probe was constructed from stainless steel hypodermic needle tubing with an outside diameter of $0.56 \mathrm{~mm}$. The catenary design allowed the ethylene trace gas injection probe to be as slender as the smallest probes reported in the cited literature, yet be operated at significantly higher flow field velocities than is capable with a cantilever configuration. Trace gas measurements in the transition duct indicate that the injection probe disturbance effects were minimal.

The flow rate of ethylene trace gas was chosen so that the bulk velocity of ethylene at the injection probe 
exit would approximately match the free stream velocity. For the transition duct experiments, with the free stream Mach number nominally equal to 0.50 , an ethylene volumetric flow rate of approximately $600 \mathrm{ml} / \mathrm{min}$ was required. The rate at which trace gas was injected was controlled with a Datametrics model 825 mass flow controller.

Ethylene trace gas was injected from the seven different locations shown in Fig. 1. All trace gas injection locations were within an axial plane situated one radius upstream of the beginning of the transition duct $(x / D=$ $-0.5)$. One injection location was coincident with the centerline $(y / D=0.0, z / D=0.0)$. The remaining six injection locations were placed radially from the centerline at $r / D=0.249$ and $r / D=0.498$ along the $z$-axis (i.e. $y / D=0.0, z / D=0.249$ and $y / D=0.0, z / D=$ 0.498 ) and along lines through the centerline $60^{\circ}$ on either side of the $z$-axis (i.e. $y / D=-0.215, z / D=0.124$; $y / D=0.215, z / D=0.124 ; y / D=-0.430, z / D=$ 0.249 and $y / D=0.430, z / D=0.249$ ).

Flow Field Sampling System

The purpose of the flow field sampling system was to provide the ethylene concentration measurement instrument with a steady and continuous supply of flow field sample from a specified location. The components of the flow field sampling system included the sampling probe or tube, the probe positioning system, and the sample pump and tubing necessary to maintain the flow of sample from the sampling probe to the ethylene concentration measurement instrument.

The most common flow field sampling probe design used in the past employed features similar to a single-hole Pitot type pressure probe. ${ }^{8,9,13}$ The flow field sample was drawn through the single probe opening. Another probe design reported was similar to a three-hole "cobra" type pressure probe. ${ }^{10}$ When using this kind of probe the

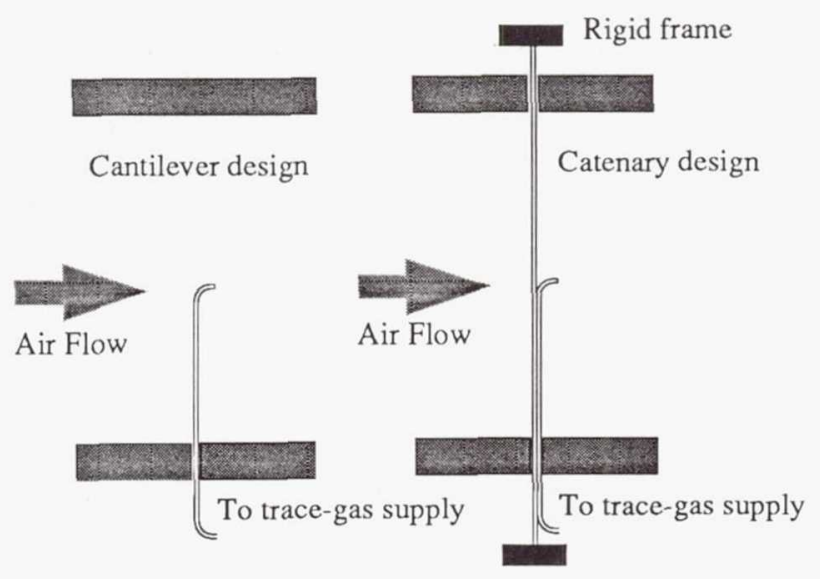

Rigid frame

Fig. 3 Cantilever and catenary injection probe designs. two side holes were used to align the yaw axis of the probe with the flow field direction while the center hole was used to draw the sample.

For the references cited above the measurements were performed at relatively low velocities, in all cases the Mach number was less than 0.10. One impediment to applying the ethylene trace gas technique to higher speed flows has been the perceived need for isokinetic sampling of the flow field (i.e. sampling at a velocity through the probe opening that matches the local host flow velocity). Isokinetic sampling is actually not necessary to accurately measure the local concentration of trace gas in a host gas flow. Injecting trace gas into the flow field creates an Eulerian concentration field. The local value of the concentration in the flow field does not depend on the rate at which the sample is acquired. However, if the sample velocity through the probe opening greatly exceeds the local host flow velocity the spatial resolution ability of the probe would deteriorate.

Another sampling consideration is the importance of aligning the sampling probe precisely in the direction of the host flow. This concern is also largely unnecessary if the proper precaution is taken. Because of the measurement technique, the concentration of the sample will not depend on the orientation of the probe when the sample is acquired, provided the presence of the probe doesn't materially alter the flow field. The sampling probe is usually the same size as a pressure probe, where the disturbance caused by the probe is usually acceptable. These principals of sampling rate and sampling probe alignment were confirmed during initial tests of the trace gas instruments and technique described in the Experimental Methods and Results section.

The locations of the trace gas concentration measurement planes are shown in Fig. 1. The distance between the probe access openings in the transition duct within each measurement plane was too large to satisfactorily resolve the expected concentration gradients with a conventional flow field sampling probe. A novel method for sampling was therefore designed to allow finer spatial resolution in the measurement planes while still using the existing probe access openings. The design employed as the sampling probe a long straight flexible tube that extended upstream to the sampling plane while firmly base mounted at a fixed location at the exit of the transition duct. The tube was constructed from stainless steel hypodermic needle tubing with outside and inside diameters of $1.83 \mathrm{~mm}$ and $1.37 \mathrm{~mm}$, respectively. The position of the tube opening was controlled by a positioning arm and the flow field sample was drawn through the tube opening.

The trace gas sampling probe and positioning arm are shown in Fig. 4. The location where the probe is mounted at the duct exit lies downstream beyond the right border of this photograph. The position of the probe opening can be visualized as lying on the surface of an imaginary scribed sphere whose radius is equal to the 


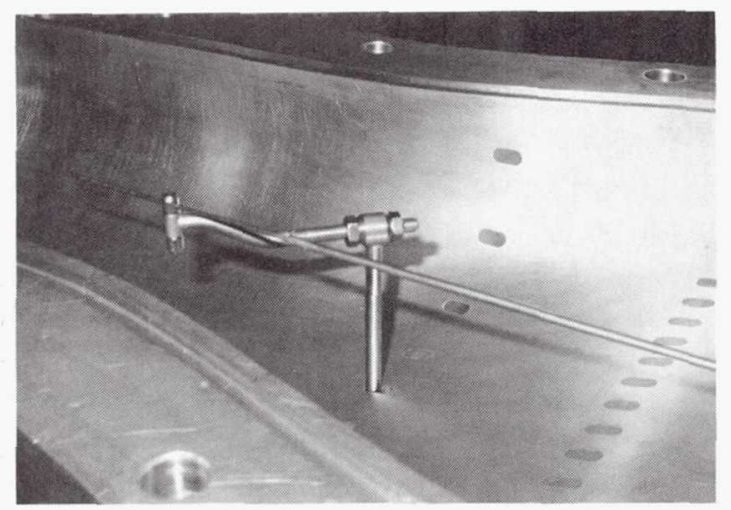

Fig. 4 Flow field sampling probe and positioning arm.

probe length. Because the probe length was sufficiently large, the deviation of the surface of the scribed sphere from the measurement plane was negligible. Different length sampling tubes were used for each measurement plane.

A sampling pump was required to supply the ethylene concentration measuring system with a continuous supply of flow field sample. This pump was necessary to overcome the pressure loss in the tubing between the sampling probe opening and the ethylene concentration measuring system and because the total pressure of the flow field was slightly less than atmospheric pressure. The sampling pump was located upstream of the ethylene concentration measuring system. A K.N.F. Neuberger, Inc., Model N05ANI pump was used. This is an oil free pump designed specifically for contamination free pumping of gases. Placing the pump upstream rather than downstream of the ethylene concentration measuring equipment avoids operation at below atmospheric pressure which allows the possibility of atmospheric air leaking into the ethylene concentration measuring equipment and diluting the sample.

\section{Ethylene Concentration Measuring System}

A GOW-MAC Instrument Company Model 23-500 Total Hydrocarbon Analyzer was used for this research project. This instrument utilized a flame ionization detector (FID) for measuring hydrocarbon concentration. The FID consists of a small burner within which fuel and oxidizer are mixed and burned. Hydrogen and zero-gas air (air containing less than $0.5 \mathrm{ppm}$ total hydrocarbons), were used as fuel and oxidizer, respectively, so there were no hydrocarbons present in this flame. When the sample was mixed with the flame, any hydrocarbons were burned and the carbon atoms became ionized. The carbon ions and electrons passed between two electrodes, decreasing the resistance between the electrodes and thus permitting an electric current to pass. This current was directly proportional to the amount of carbon ions present.

The FID responds to both the amount of carbon atoms present in the sample, and the rate at which the sample passes through the detector. This makes it very important to maintain a constant flow rate of sample through the FID. Hardware within the total hydrocarbon analyzer maintained a constant specified flow rate into the FID regardless of the sample flow rate into the total hydrocarbon analyzer, provided, of course, the flow rate into the total hydrocarbon analyzer exceeded the desired flow rate to the FID. The fraction of sample fluid not directed to the FID was discharged to the atmosphere. Therefore, fluctuations in the flow rate of the trace gas sampling pump did not affect the flow rate into the FID. The suggested flow rate of sample fluid into the FID specified by the manufacturer, $25 \mathrm{ml} / \mathrm{min}$, was used.

\section{Experimental Methods and Results}

\section{Test Conditions}

An aerodynamic survey of the flow field one radius upstream of the transition duct inlet $(x / D=-0.5)$ was conducted to establish test conditions. The centerline Mach number was 0.50 . The Reynolds number, based on the centerline velocity and the transition duct inlet diameter, was $2,086,000$. The boundary layer thickness was approximately 4 percent of the inlet diameter of the transition duct and the boundary layer shape factor was 1.42 , confirming turbulent flow.

\section{Trace Gas Measurements}

The flame ionization detector was calibrated by preparing a mixture of ethylene and zero-gas air of known concentration and supplying this as a sample to the total hydrocarbon analyzer. This calibration was performed periodically throughout the period of trace gas testing. After the initial calibration, it was seldom necessary to make any further adjustments.

The concentration measured in trace gas surveys was the mean ethylene mole fraction, given by Eq. (2). In Eq. (2), $n_{\text {ethylene }}$ refers to the molar amount of ethylene present in a sample and $n$ refers to the total molar amount of the quantity of matter in the sample. The results are presented as a mean ethylene concentration coefficient $C^{*}$, where the local mean ethylene concentration has been normalized by the peak value of mean ethylene concentration measured for that particular distribution, as shown in Eq. (3).

$$
\begin{gathered}
C_{\text {ethylene }}=\frac{n_{\text {ethylene }}}{n} \\
C^{*}=\frac{C_{\text {ethylene }}}{C_{\text {ethylene,peak }}}
\end{gathered}
$$

Prior to conducting the transition duct experiments, preliminary trace gas measurements were made in a calibration jet to insure that the trace gas equipment was operating properly. These measurements were also performed 
to reveal information about the trace gas technique itself. A test was performed to determine the sensitivity of the ethylene concentration measurements to the ethylene injection rate. Ethylene was injected at the centerline of the calibration tunnel jet. Two separate cross stream sample surveys were conducted approximately $110 \mathrm{~mm}$ downstream of the injection point. The rate of ethylene injection varied between the two surveys while all other test conditions were unchanged. A comparison of the measured concentration distributions presented as concentration coefficients showed very little difference. Within some reasonable limitations, the concentration coefficient appears to be insensitive to the rate at which ethylene is injected into the host flow.

To test the sensitivity of the ethylene concentration measurements to a mismatch between the sample velocity at the probe opening and the host flow velocity, two cross stream sample surveys were compared. For both surveys ethylene was injected at the centerline of the calibration jet and the flow was sampled in cross stream planes approximately $110 \mathrm{~mm}$ downstream of the point of injection. In one case the jet Mach number was approximately 0.10 . and in the second case the jet Mach number was approximately 0.60 . The rate at which the sample was drawn was held constant, insuring a significant velocity mismatch between these two cases. In both cases the sample velocity did not exceed the host flow velocity. The results showed little difference in the concentration coefficient, demonstrating that isokinetic sampling is not necessary.

To test the sensitivity of the ethylene concentration measurements to the sampling probe alignment, an Lshaped type sampling probe was employed. This probe was constructed with the probe opening in line with the probe shaft axis so that the probe could be rotated, changing the probe orientation, without changing the location of the probe opening. Thus, this probe could be intentionally misaligned from the host flow direction without changing the probe opening location. Ethylene was injected at the jet centerline and measurements were made downstream at a single location. The probe alignment was changed approximately $30^{\circ}$ on either side of the host flow direction without any discernible change in the measured sample concentration. This evidence suggests, that at least within this range of angles, concentration measurements are insensitive to misalignment of the probe with the host flow.

The trace gas measurements in the transition duct were made following the preliminary tests described above. Twenty-eight separate planer concentration distributions resulted from introducing ethylene at the seven injection locations and measuring the resulting concentration field at the four cross stream measurement planes. The results for each of the 28 separate planer concentration distributions were obtained by recording the ethylene concentration at approximately 100 locations within the measurement plane. Ten separate ethylene concentration measurements were recorded at each location and the mean of these ten values was used for plotting and analysis. The 28 measured concentration distributions are shown in Fig. 5. The contour levels shown in Fig. 5 are at $20,40,60$, and 80 percent of the peak value.

The axial locations of the four ethylene concentration measurement planes and the measurement planes for the aerodynamic measurements reported in Ref. 7 were not the same. This was because of the difference in hardware required for each method. Despite the different locations, at three of the trace gas measurement planes it was possible to obtain aerodynamic data, either directly (trace gas plane 3 and aerodynamic plane 2 were at identical locations, while trace gas plane 2 and aerodynamic plane 1 were very close) or by interpolation (trace gas plane 4 was between aerodynamic planes 3 and 4). Fig. 5 also shows the transverse components of velocity superimposed over the trace gas concentration contours. No aerodynamic measurements were available at trace gas plane 1 .

\section{Discussion and Conclusions}

Eq. (4) governs the transport of trace gas in a turbulent incompressible flow. The left hand side of Eq. (4) represents the convection of the mean concentration of trace gas by the mean flow, the first term on the right hand side represents the molecular diffusion of the mean trace gas concentration and the second term on the right hand side represents the turbulent diffusion of the trace gas concentration.

$$
U_{i} \frac{\partial C}{\partial x_{i}}=D_{m} \frac{\partial}{\partial x_{i}}\left(\frac{\partial C}{\partial x_{i}}\right)-\frac{\partial\left(\overline{u_{i}^{\prime} c^{\prime}}\right)}{\partial x_{i}}
$$

Applying mixing length theory for homogeneous turbulence, the turbulent diffusion tensor can be written in terms of a symmetric tensor. Further, the molecular diffusion term is much smaller than the turbulent diffusion term. Under these conditions, Eq. (4) can be expressed as Eq. (5).

$$
U_{i} \frac{\partial C}{\partial x_{i}}=\frac{\partial}{\partial x_{i}}\left(D_{i j} \frac{\partial C}{\partial x_{j}}\right)
$$

When the trace gas technique is used to infer information about the flow field there is an ambiguity problem. The ambiguity problem arises when experimental measurements, which represent a solution to Eq. (5) with a known boundary condition, are used to determine the coefficients of Eq. (5), $U_{i}$ and $D_{i j}$, which are unknown. Although the solutions to Eq. (5), with some restrictions on the coefficients and boundary conditions, are unique, Eq. (5) with different coefficients and identical boundary conditions can give rise to identical solutions. The ambiguity problem is exacerbated by the fact that the experimental data are not known everywhere, but only at a 


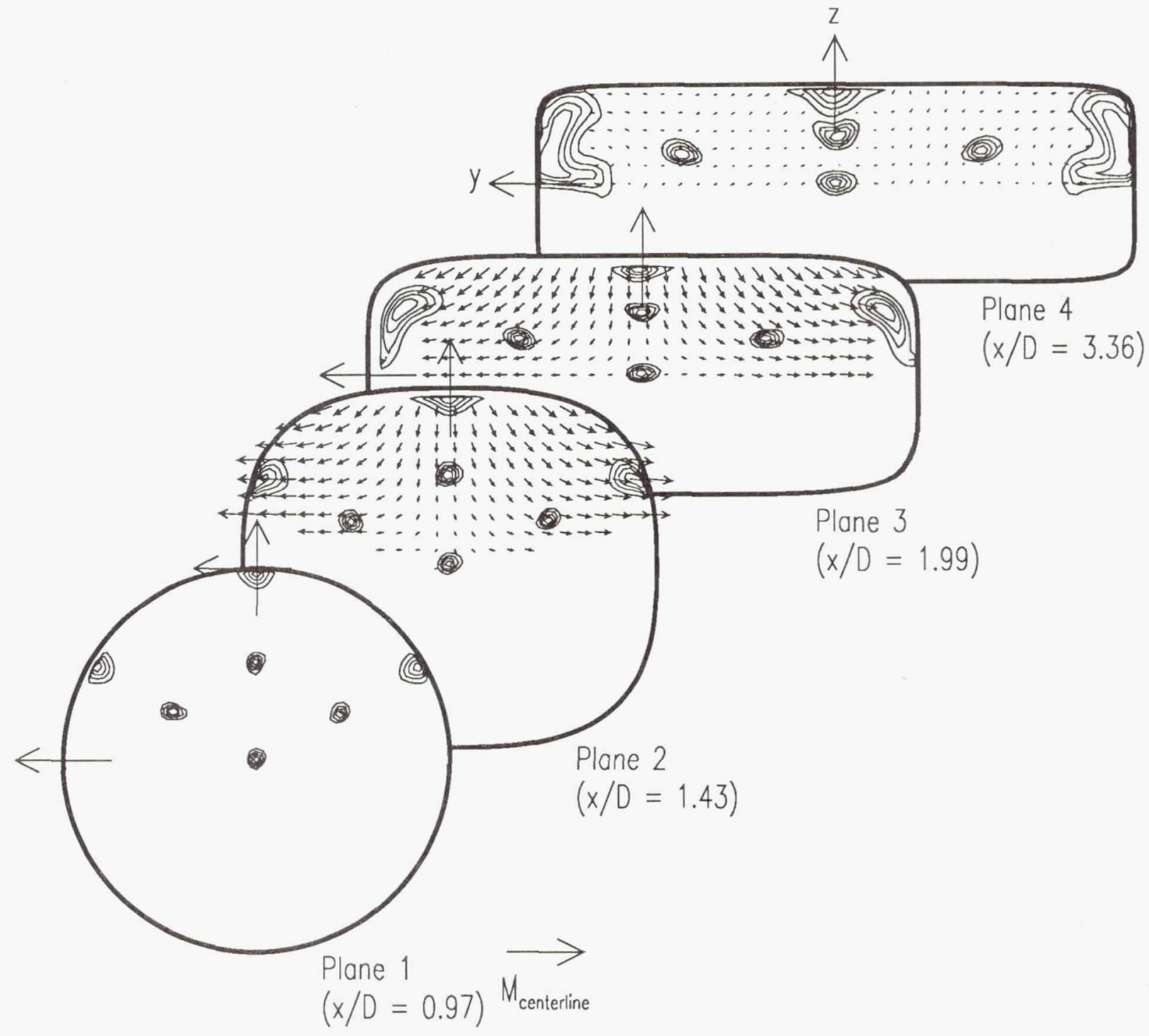

Fig. 5 Concentration contours and transverse velocity components.

finite number of locations where measurements are made. This makes it difficult to interpret trace gas results without either making some assumptions which simplify Eq. (5), or supplementing the trace gas data with additional information, or both. The supplemental information may be experimental data or numerical results.

The following analysis will consider one special case of the turbulent transport equation, when the molecular diffusion is negligible, the turbulent diffusion is homogeneous but not necessarily isotropic, the turbulent diffusion in the axial direction is negligible, and the mean flow is uniform and in the $x$ direction. Under these restrictions Eq. (5), an elliptic partial differential equation, reduces to Eq. (6), a parabolic partial differential equation like the heat equation, where the $x$ dimension in Eq. (6) cor- responds to time in the heat equation. Without any loss in generality, it is assumed the $y$ - and $z$-axes are coincident with the principal axes of the tensor $D_{i j}$, so that $D_{y z}=0$.

$$
\begin{gathered}
U \frac{\partial C}{\partial x}=D_{y y} \frac{\partial^{2} C}{\partial y^{2}}+2 D_{y z} \frac{\partial}{\partial y}\left(\frac{\partial C}{\partial z}\right)+D_{z z} \frac{\partial^{2} C}{\partial z^{2}} \\
C=\frac{S U}{4 \pi x \sqrt{D_{y y} D_{z z}}} \exp -\frac{U}{4 x}\left(\frac{y^{2}}{D_{y y}}+\frac{z^{2}}{D_{z z}}\right) \\
\sigma_{y}^{2}=\frac{2 x D_{y y}}{U} \quad \sigma_{z}^{2}=\frac{2 x D_{z z}}{U}
\end{gathered}
$$




\begin{tabular}{|c|c|c|c|c|c|c|c|c|}
\hline \multirow{2}{*}{$\begin{array}{c}\text { Sampling } \\
\text { location } \\
\frac{x}{D}\end{array}$} & \multicolumn{2}{|c|}{ Peak value location } & \multicolumn{2}{|c|}{ Mean value location } & \multicolumn{2}{|c|}{ Variance } & \multirow{2}{*}{$\begin{array}{c}\text { Covariance } \\
\qquad \begin{array}{c}\frac{\sigma_{y z}}{D^{2}} \\
\left(\times 10^{4}\right)\end{array}\end{array}$} & \multirow{2}{*}{$\begin{array}{c}\text { Area } \\
\text { estimate }\end{array}$} \\
\hline & $\begin{array}{c}\frac{y}{D} \\
(\times 100)\end{array}$ & $\begin{array}{c}\frac{z}{D} \\
(\times 100) \\
\end{array}$ & $\begin{array}{c}\frac{\mu_{y}}{D} \\
(\times 100) \\
\end{array}$ & $\begin{array}{c}\frac{\mu_{z}}{D} \\
(\times 100) \\
\end{array}$ & $\begin{array}{c}\frac{\sigma_{y}^{2}}{D^{2}} \\
\left(\times 10^{4}\right)\end{array}$ & $\begin{array}{c}\frac{\sigma_{x}^{2}}{D^{2}} \\
\left(\times 10^{4}\right)\end{array}$ & & \\
\hline 0.97 & 0.0 & 0.1 & -0.1 & 0.4 & 2.2 & 3.2 & 0.0 & 8.3 \\
\hline 1.43 & 0.0 & 1.2 & -0.2 & 1.2 & 2.8 & 2.5 & 0.4 & 8.3 \\
\hline 1.99 & 0.0 & 0.7 & 0.0 & 0.4 & 5.3 & 1.8 & 0.3 & 9.6 \\
\hline 3.36 & 0.1 & -0.1 & 0.4 & 0.2 & 7.4 & 3.0 & 0.0 & 14.8 \\
\hline
\end{tabular}

Table 1 Concentration distribution statistics for injection at $y / D=0.0, z / D=0.0$

For the case of a point source of trace gas located at the origin, the solution to Eq. (6) is given by Eq. (7). The variances of the solution are given by Eq. (8), the covariance is zero because of the choice of axes. Although Eq. (6) appears simplified beyond the point of relevance, its solution provides some useful insight into interpreting trace gas results. For this particular solution:

(1) The concentration contours will always be concentric ellipses.

(2) The variances will always increase with axial distance.

(3) The area of the trace gas spreading can be estimated by Eq. (9) which is the area of the ellipse defined by Eq. (10).

$$
\begin{gathered}
\text { Area }=\pi \sqrt{\sigma_{y}^{2} \sigma_{z}^{2}-\sigma_{y z}^{2}} \\
\frac{y^{2} \sigma_{z}^{2}}{\sigma_{y}^{2} \sigma_{z}^{2}-\sigma_{y z}^{2}}-\frac{2 y z \sigma_{y z}}{\sigma_{y}^{2} \sigma_{z}^{2}-\sigma_{y z}^{2}}+\frac{z^{2} \sigma_{y}^{2}}{\sigma_{y}^{2} \sigma_{z}^{2}-\sigma_{y z}^{2}}=1
\end{gathered}
$$

For each of the 28 measured concentration distributions, the mean values, variances, and covariance of the concentration distributions were calculated. The definitions of these statistics are given in Eqs. (11) through (14). These values are presented in Tables 1 through 5 . Also presented in these tables are the peak value location and an estimate of the spreading area based on Eq. (9). These statistics are useful to help quantify characteristics of the trace gas measurements. The variances measure the amount of trace gas spreading in the $y$ and $z$ directions while the covariance value indicates the orientation of the contours. All values in the tables are nondimensional.

$$
\begin{gathered}
E[\phi]=\frac{\int \phi C d A}{\int C d A}, \phi=y, z, y^{2}, y z, z^{2} \\
\mu_{y}=E[y], \mu_{z}=E[z] \\
\sigma_{y}^{2}=E\left[\left(y-\mu_{y}\right)^{2}\right], \sigma_{z}^{2}=E\left[\left(z-\mu_{z}\right)^{2}\right] \\
\sigma_{y z}=E\left[\left(y-\mu_{y}\right)\left(z-\mu_{z}\right)\right]
\end{gathered}
$$

For trace gas injected at the transition duct centerline, $y / D=0.0, z / D=0.0$, the covariance, Table 1 , remained relatively small along the length of the duct.

\begin{tabular}{|c|c|c|c|c|c|c|c|c|}
\hline \multirow{2}{*}{$\begin{array}{c}\text { Sampling } \\
\text { location } \\
\frac{x}{D}\end{array}$} & \multicolumn{2}{|c|}{ Peak value location } & \multicolumn{2}{|c|}{ Mean value location } & \multicolumn{2}{|c|}{ Variance } & \multirow{2}{*}{$\begin{array}{c}\text { Covariance } \\
\qquad \begin{array}{c}\frac{\sigma_{y z}}{D^{2}} \\
\left(\times 10^{4}\right)\end{array}\end{array}$} & \multirow{2}{*}{$\begin{array}{c}\text { Area } \\
\text { estimate }\end{array}$} \\
\hline & $\begin{array}{c}\frac{y}{D} \\
(\times 100)\end{array}$ & $\begin{array}{c}\frac{z}{D} \\
(\times 100)\end{array}$ & $\begin{array}{c}\frac{\mu_{y}}{D} \\
(\times 100)\end{array}$ & $\begin{array}{c}\frac{\mu_{z}}{D} \\
(\times 100)\end{array}$ & $\begin{array}{c}\frac{\sigma_{y}^{2}}{D^{2}} \\
\left(\times 10^{4}\right)\end{array}$ & $\begin{array}{c}\frac{\sigma_{\frac{2}{2}}^{2}}{D^{2}} \\
\left(\times 10^{4}\right)\end{array}$ & & \\
\hline 0.97 & -0.1 & 25.7 & -0.2 & 25.3 & 2.1 & 3.5 & 0.2 & 8.4 \\
\hline 1.43 & 0.0 & 23.9 & -0.4 & 24.3 & 3.3 & 2.5 & 0.2 & 9.0 \\
\hline 1.99 & 0.0 & 15.9 & 0.0 & 16.5 & 5.6 & 2.0 & -0.1 & 10.4 \\
\hline 3.36 & 1.5 & 11.8 & 0.5 & 13.0 & 9.9 & 4.9 & -0.1 & 21.8 \\
\hline
\end{tabular}
This indicates that the turbulence field is symmetric along the duct centerline. These trace gas contours became increasingly elongated in the $y$ direction at downstream locations. This can be seen in the magnitude of $\sigma_{y}^{2} / D^{2}$ relative to $\sigma_{z}^{2} / D^{2}$. Two possible explanations for this

Table 2 Concentration distributions statistics for injection at $y / D=0.0, z / D=0.249$ 


\begin{tabular}{ccccccccc}
\hline $\begin{array}{c}\text { Sampling } \\
\text { location }\end{array}$ & \multicolumn{2}{c}{ Peak value location } & \multicolumn{2}{c}{ Mean value location } & Variance & Covariance & $\begin{array}{c}\text { Area } \\
\text { estimate }\end{array}$ \\
$\frac{x}{D}$ & $\frac{y}{D}$ & $\frac{z}{D}$ & $\frac{\mu_{y}}{D}$ & $\frac{\mu_{z}}{D}$ & $\frac{\sigma_{y}^{2}}{D^{2}}$ & $\frac{\sigma_{z}^{2}}{D^{2}}$ & $\frac{\sigma_{y z}}{D^{2}}$ & $\left(\times 10^{4}\right)$ \\
& $(\times 100)$ & $(\times 100)$ & $(\times 100)$ & $(\times 100)$ & $\left(\times 10^{4}\right)$ & $\left(\times 10^{4}\right)$ & $\left(\times 10^{4}\right)$ \\
\hline 0.97 & -0.1 & 49.4 & -0.1 & 47.3 & 6.2 & 2.5 & -0.1 & 12.3 \\
1.43 & 0.0 & 44.1 & -0.2 & 42.4 & 23.3 & 2.3 & -0.3 & 22.8 \\
1.99 & 0.0 & 28.4 & -0.4 & 26.9 & 40.3 & 1.5 & 0.1 & 24.6 \\
3.36 & 0.0 & 25.0 & -0.3 & 22.4 & 50.7 & 4.7 & 0.0 & 48.7 \\
\hline \hline
\end{tabular}

Table 3 Concentration distributions statistics for injection at $y / D=0.0, z / D=0.498$

are either the turbulence is anisotropic, that is $\overline{v^{\prime 2}}>\overline{w^{\prime 2}}$, or the concentration distribution is being affected by the transverse components of velocity. Two experimental facts suggest that the elongation is produced by the transverse velocity components. First, the value of $\sigma_{z}^{2} / D^{2}$ is decreasing from plane 1 to plane 3 and then increases from plane 3 to plane 4 . It would be impossible for $\sigma_{z}^{2} / D^{2}$ to decrease by turbulent diffusion alone in a uniform flow field. Second, velocity measurements show flow converging in the $x z$-plane and diverging in the $x y$ plane. The region of decreasing $\sigma_{z}^{2} / D^{2}$ corresponds to the region where the transverse velocity components are greatest. This can be seen in Fig. 5 at planes 2 and 3 . At plane 4 of Fig. 5, the transverse velocity components near the centerline are negligible and $\sigma_{z}^{2} / D^{2}$ has increased between planes 3 and 4 .

For trace gas injected at $y / D=0.0, z / D=0.249$ (12 o'clock, halfway between the duct centerline and the wall) the statistical results, Table 2 , are similar to the results for trace gas injected at $y / D=0.0, z / D=0.0$.
However, at planes 3 and 4 the contour shapes for the two injection locations, Fig. 5, appear to be noticeably different. The contour shapes for trace gas injected at $y / D=0.0, z / D=0.249$ are no longer elliptic, rather they are bean shaped, having only one plane of symmetry. The simple model of homogeneous turbulence in a uniform mean flow allows only elliptic shaped contours, which would suggest that these contours result from turbulence inhomogeneity, or from nonuniformity in the mean flow. The mean flow certainly is nonuniform. However, there is nothing to indicate that the turbulence is not homogeneous. Thus, the bean shape of the contours probably results from transverse velocity components. Also, the amount of spreading is approximately the same as for trace gas injected at $y / D=0.0, z / D=0.0$, except at plane 4 , which indicates roughly the same turbulence conditions in both regions of flow.

For trace gas injected at $y / D=0.0, z / D=0.498$ (12 o'clock, near the duct wall) the value of $\sigma_{y}^{2} / D^{2}$ in Table 3 indicates a very large elongation in the $y$ direc-

\begin{tabular}{|c|c|c|c|c|c|c|c|c|}
\hline \multirow{2}{*}{$\begin{array}{c}\text { Sampling } \\
\text { location } \\
\frac{x}{D}\end{array}$} & \multicolumn{2}{|c|}{ Peak value location } & \multicolumn{2}{|c|}{ Mean value location } & \multicolumn{2}{|c|}{ Variance } & \multirow{2}{*}{$\begin{array}{c}\text { Covariance } \\
\qquad \begin{array}{c}\frac{\sigma_{y z}}{D^{2}} \\
\left(\times 10^{4}\right)\end{array}\end{array}$} & \multirow{2}{*}{$\begin{array}{c}\text { Area } \\
\text { estimate } \\
\left(\times 10^{4}\right)\end{array}$} \\
\hline & $\begin{array}{c}\frac{y}{D} \\
(\times 100)\end{array}$ & $\begin{array}{c}\frac{z}{D} \\
(\times 100)\end{array}$ & $\begin{array}{c}\frac{\mu_{y}}{D} \\
(\times 100)\end{array}$ & $\begin{array}{c}\frac{\mu_{z}}{D} \\
(\times 100)\end{array}$ & $\begin{array}{c}\frac{\sigma_{y}^{2}}{D^{2}} \\
\left(\times 10^{4}\right)\end{array}$ & $\begin{array}{c}\frac{\sigma_{z}^{2}}{D^{2}} \\
\left(\times 10^{4}\right)\end{array}$ & & \\
\hline \multirow{2}{*}{0.97} & -21.7 & 12.1 & -21.4 & 12.6 & 3.9 & 1.8 & -0.6 & 8.2 \\
\hline & 21.8 & 12.5 & 21.4 & 12.4 & 3.0 & 3.0 & 0.9 & 8.9 \\
\hline \multirow{2}{*}{1.43} & -25.8 & 11.6 & -25.7 & 12.1 & 3.2 & 2.6 & -0.6 & 8.8 \\
\hline & 25.6 & 12.7 & 25.6 & 12.7 & 3.0 & 2.4 & 0.8 & 8.0 \\
\hline \multirow{2}{*}{1.99} & -32.2 & 9.7 & -32.0 & 9.5 & 5.3 & 2.2 & -1.0 & 10.3 \\
\hline & 32.2 & 9.4 & 32.5 & 9.4 & 5.9 & 1.8 & 0.6 & 10.2 \\
\hline \multirow{2}{*}{3.36} & -40.8 & 8.2 & -39.9 & 8.2 & 8.4 & 3.3 & -1.4 & 15.8 \\
\hline & 38.3 & 8.1 & 38.2 & 8.7 & 8.2 & 3.5 & 1.7 & 16.0 \\
\hline
\end{tabular}

Table 4 Concentration distributions statistics for injection at $y / D=-0.215, z / D=0.124$ and $y / D=0.215, z / D=0.124$ 


\begin{tabular}{ccccccccc}
\hline $\begin{array}{c}\text { Sampling } \\
\text { location } \\
\frac{x}{D}\end{array}$ & Peak value location & \multicolumn{2}{c}{ Mean value location } & \multicolumn{2}{c}{ Variance } & Covariance & $\begin{array}{c}\text { Area } \\
\text { estimate }\end{array}$ \\
& $\begin{array}{c}\frac{y}{D} \\
(\times 100)\end{array}$ & $\frac{z}{D}$ & $\frac{\mu_{y}}{D}$ & $\frac{\mu_{z}}{D}$ & $\frac{\sigma_{y}^{2}}{D^{2}}$ & $\frac{\sigma_{z}^{2}}{D^{2}}$ & $\frac{\sigma_{y z}}{D^{2}}$ & \\
$(\times 100)$ & $(\times 100)$ & $(\times 100)$ & $\left(\times 10^{4}\right)$ & $\left(\times 10^{4}\right)$ & $\left(\times 10^{4}\right)$ & $\left(\times 10^{4}\right)$ \\
\hline \multirow{2}{*}{0.97} & -42.1 & 24.1 & -39.6 & 23.4 & 4.1 & 5.5 & 1.0 & 14.7 \\
& 42.2 & 24.7 & 40.3 & 23.2 & 3.9 & 4.8 & -0.8 & 13.4 \\
1.43 & -46.6 & 21.9 & -46.6 & 23.0 & 6.9 & 7.1 & 2.6 & 20.5 \\
& 49.9 & 21.6 & 46.3 & 22.9 & 6.9 & 6.9 & -2.4 & 20.3 \\
1.99 & -62.4 & 17.1 & -61.2 & 15.9 & 17.8 & 25.9 & 12.4 & 55.0 \\
& 62.5 & 17.2 & 62.9 & 14.5 & 17.3 & 25.1 & -13.1 & 50.4 \\
3.36 & -72.7 & 12.8 & -69.0 & 9.7 & 24.1 & 55.1 & -5.4 & 113.2 \\
& 72.6 & 14.9 & 69.1 & 9.9 & 25.0 & 53.8 & 5.1 & 114.2 \\
\hline \hline
\end{tabular}

Table 5 Concentration distributions statistics for injection at

$y / D=-0.430, z / D=0.249$ and $y / D=0.430, z / D=0.249$

tion. The results of Klebanoff ${ }^{14}$ show that the magnitude of the transverse turbulent velocity fluctuations, and turbulent viscosity coefficient, increase greatly in a turbulent boundary layer. In a turbulent boundary layer the turbulence is very heterogeneous. The turbulence decreases rapidly away from the duct surface, in this case the $z$ direction, while retaining its magnitude in directions parallel to the wall, specifically the $y$ direction. This should lead to preferential diffusion in the $y$ direction. However, turbulent diffusion alone is not exclusively responsible for the observed contours because $\sigma_{z}^{2} / D^{2}$ again decreases from plane 1 to plane 3 . The total amount of spreading is roughly twice that observed of trace gas injected at $y / D=0.0, z / D=0.0$ and $y / D=0.0, z / D=0.249$. Surface oil film visualization results reported in Ref. 7 show large transverse velocities in this region. It appears that both turbulent diffusion and transverse velocities are responsible for the large transport of trace gas in the $y$ direction displayed by the contours.

For trace gas injected at $y / D=-0.215, z / D=$ 0.124 and $y / D=0.215, z / D=0.124$ (about 10 and 2 o'clock, between the duct centerline and wall) the amount of spreading at each of the four planes is comparable to the spreading of trace gas injected at $y / D=0.0, z / D=$ 0.0 and $y / D=0.0, z / D=0.249$. This indicates that outside the boundary layer the turbulence is nominally homogeneous. Each contour becomes more elongated as it travels downstream. Unlike the results for trace gas injected at $y / D=0.0, z / D=0.0$ and $y / D=$ $0.0, z / D=0.249$, the contours change their orientation. This can be observed in the covariance values. The major axes of the contours are no longer parallel to the $y$-axis, but are inclined to the $y$-axis. This probably results from the transverse velocities.

Trace gas injected at $y / D=-0.430, z / D=0.249$ and $y / D=0.430, z / D=0.249$ (about 10 o'clock and 20 'clock, near the duct wall) produced the most interesting results. At planes 1 and 2 the statistics, Table 5, are nearly identical to the statistics for trace gas injected at $y / D=0.0, z / D=0.498$, except for the orientation. At plane 2, however, the contours in Fig. 5 appear visibly different, assuming a cusped teardrop shape. At plane 3 the statistics differ significantly from the statistics of trace gas injected at $y / D=0.0, z / D=0.498$. The spreading was approximately twice as great. This resulted from the transfer by axial vortices of boundary layer fluid, with its greater turbulence intensity and diffusion, to a large area near the side walls. At plane 4 the much greater spreading by vortices is obvious. The unusual contour shape results from pairs of counter-rotating vortices near the transition duct side walls. These vortices were identified and discussed in detail in Ref. 7. The total pressure coefficient contours of Fig. 6 clearly indicate large regions of lower total pressure near the duct side walls. The data of Figs. 5 and 6 , along with the results of the statistical analyses, confirm that the convection of boundary layer fluid by the sidewall vortices and turbulent diffusion are responsible for these large regions of lower total pressure.

\section{Summary}

The ethylene trace gas technique provided an accurate Lagrangian flow field representation for high velocity flow within the transition duct. These data are useful for assessing the accuracy of CFD codes used to predict the transport and mixing of a nonreacting gas. The methods developed to obtain the ethylene trace gas data can be used in other experimental configurations with very little modification. In fact, this technique is presently being used by Davis et al. ${ }^{15}$ to measure the mixing of two su- 


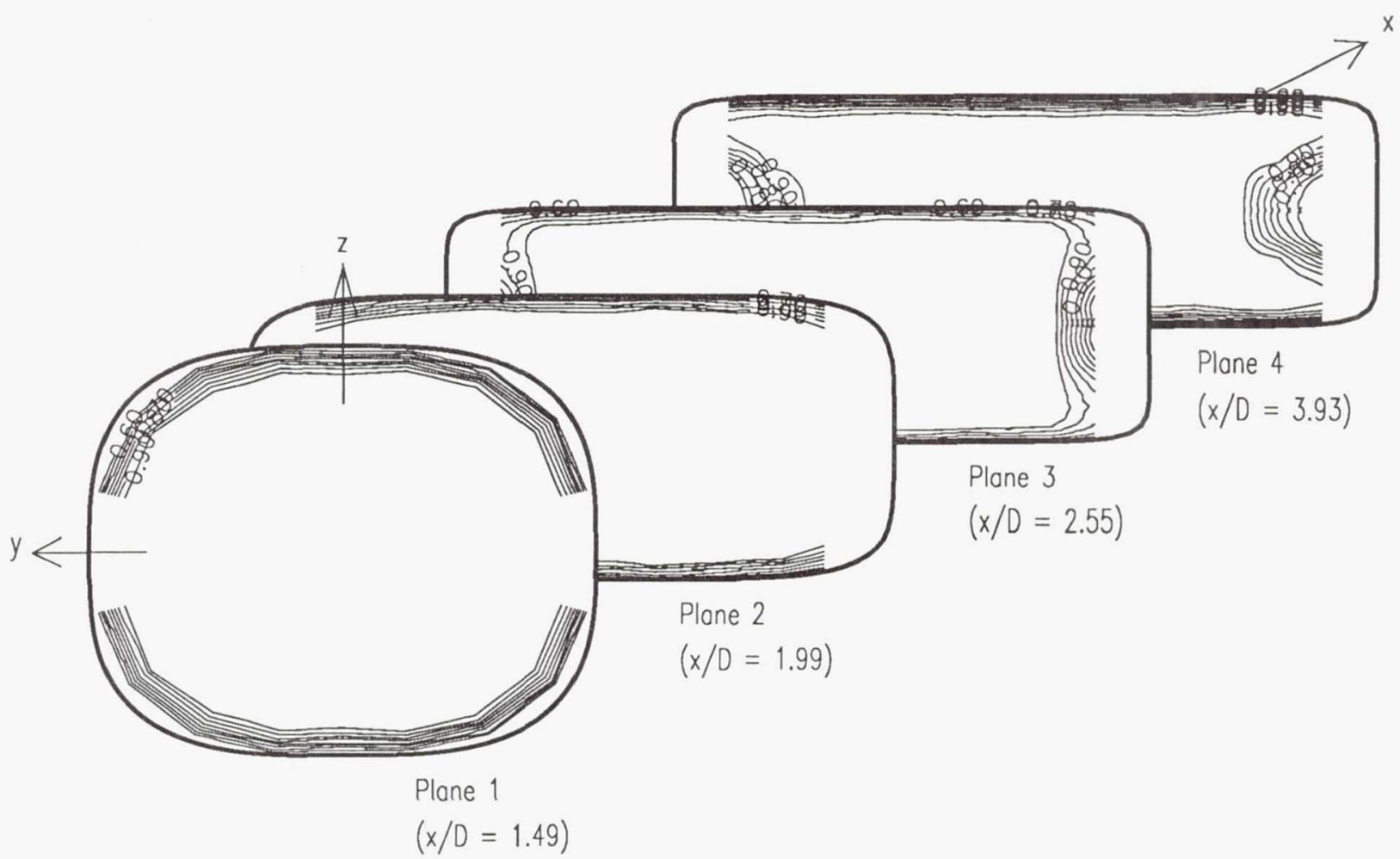

Fig. 6 Total pressure coefficient contours.

personic streams in a study of mixing nozzles for scramjet applications. The statistical methods used to help interpret the trace gas data provided a reliable way to quantify and compare contour characteristics such as shape, orientation and the amount of contour spreading. Trace gas data, along with aerodynamic and surface flow visualization data, confirmed the strong convection of wall boundary layer fluid by vortices to form significant regions of high total pressure loss in the duct.

\section{References}

${ }^{1}$ Stevens, H., Thayer, E. B., and Fullerton, J. F., "Development of the Multi-Function 2-D/C-D Nozzle," AIAA Paper 81-1491, 1981.

${ }^{2}$ Patrick, W. P. and McCormick, D. C., "Laser Velocimeter and Total Pressure Measurements in Circularto-Rectangular Transition Ducts," United Technologies Research Center Report 87-41, June 1988.

${ }^{3}$ Patrick, W. P. and McCormick, D. C., "Circular-toRectangular Duct Flows - A Benchmark Experimental Study," Society of Automotive Engineers Paper 871776, 1988.
${ }^{4}$ Miau, J. J., Lin, S. A., Chou, J. H., Wei, C.' ${ }^{\prime}$., and Lin, C. K., "An Experimental Study of Flow in a Circular-Rectangular Transition Duct," AIAA Paper 883029, 1988.

${ }^{5}$ Miau, J. J., Leu, T. S., Chou, J. H., Lin, S. A., and Lin, C. K., "Flow Distortion in a Circular-to-Rectangular Transition Duct," AIAA Journal, Vol. 28, Aug. 1990, pp. 1447-1456.

${ }^{6}$ Davis, D. O. and Gessner, F. B., "Experimental Investigation of Turbulent Flow Through a Circular-toRectangular Transition Duct," AIAA Paper 90-1505, 1990.

${ }^{7}$ Reichert, B. A., Hingst, W. R., and Okiishi, T. H., "An Experimental Comparison of Nonswirling and Swirling Flow in a Circular-to-Rectangular Transition Duct," AIAA Paper 91-0342, 1991. (also NASA TM 104359).

${ }^{8}$ Smith, B. L., "Detection of Secondary Flow in a Turbine Cascade Using a Tracer Gas Technique," M.S. Thesis, Virginia Polytechnic Institute and State University, Blacksburg, Virginia, Feb. 1983.

${ }^{9}$ Moore, J., "Flow Trajectories, Mixing, and Entropy Fluxes in a Turbine Cascade," Viscous Effects in Tur- 
bomachinary, AGARD, 1983, pp. 5-1 - 5-14. AGARD CP-351.

${ }^{10}$ Gallimore, S. J., Spanwise Mixing in Multi-Stage Axial Compressors, Ph.D. Dissertation, University of Cambridge, Cambridge, England, Sept. 1985.

${ }^{11}$ Gallimore, S. J., "Spanwise Mixing in Multistage Axial Flow Compressors: Part 2 - Throughflow Calculations Including Mixing," ASME Paper 86-GT-21, 1986.

${ }^{12}$ Moore, J., Moore, J. G., and Adhye, R. Y., "Testing Numerical Methods for Turbomachinary Flows Using Ethylene Injection Studies," Numerical Methods in Laminar and Turbulent Flows, edited by C. Taylor, W. G. Habashi, and M. M. Hafez, Pineridge Press, Swansea, U. K., 1987, pp. 1675-1686.

${ }^{13}$ Wisler, D. C., Bauer, R. C., and Okiishi, T. H., "Secondary Flow, Turbulent Diffusion and Mixing in AxialFlow Compressors," ASME Journal of Turbomachinery, Vol. 109, Oct. 1987, pp. 455-482.

${ }^{14}$ Klebanoff, P. S., "Characteristics of Turbulence in a Boundary Layer with Zero Pressure Gradient," NACA Technical Note 3178, 1954.

${ }^{15}$ Davis, D. O., Hingst, W. R., and Porro, A. R., "Experimental Investigation of a Single Flush-Mounted Hypermixing Nozzle," AIAA Paper 90-5240, 1990. (also NASA TM 103726). 


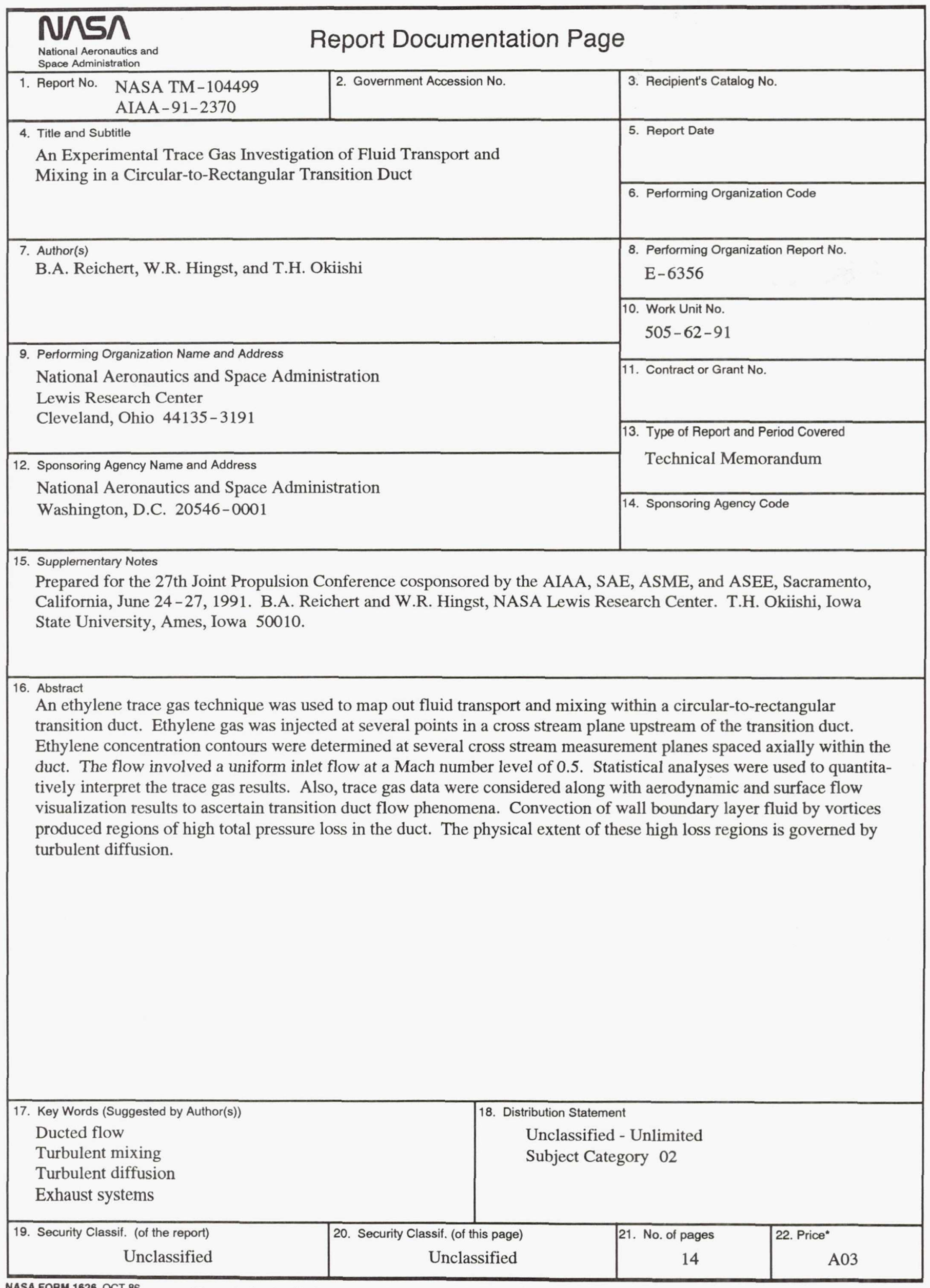

